\title{
Retinal haemorrhages segmentation using improved toboggan segmentation algorithm in diabetic retinopathy images.
}

\author{
S. Karkuzhali*, D. Manimegalai \\ Department of Information Technology, National Engineering College, Kovilpatti, Tamil Nadu, India
}

\begin{abstract}
This paper presents a new approach to segment pre-retinal and sub-retinal haemorrhage from colour fundus retinal image based on improved toboggan segmentation. Effective segmentation of pre-retinal, sub-retinal, small/blot haemorrhage detection is important in the development of screening system of Diabetic Retinopathy (DR). The strategy involves the application of edge detection and super-pixel based toboggan segmentation. The main advantage of the proposed work is that it does not depend on the manually selected parameters. This haemorrhages detection technique leads to high accuracy of $93.21 \%$ in comparison with the ground truth images from ophthalmologist.
\end{abstract}

Keywords: Haemorrhage, Retina, Toboggan segmentation, Colour fundus images, Diabetic retinopathy.

\section{Introduction}

According to the World Health Organization (WHO), approximately $28.5 \%$ and $4.4 \%$ of diabetic patients above the age of $40 \mathrm{y}$ from the year 2005 to 2008 were diagnosed with Diabetic Retinopathy (DR) and advanced DR, which can lead to blindness [1]. DR is a sight-threatening disease for diabetes patients which rupture the small blood vessels in the retina, the nerve layer in the posterior part of the eye. Early stages of DR are often symptomless. Early diagnosis and timely treatment are essential to prevent the significant vision loss from DR. Ophthalmologists are analysing diabetes patient's twodimensional fundus images for their investigation. So, every patient who is at risk of diabetes should have their eyes examined every year [2]. However, with a large number of retinal diseases affected patients, paucity in number of ophthalmologist and expenditure of current hospital-based system are the major barricades to achieve recommended screening system. Developing the automated Computer Aided Diagnosis (CAD) tool can assist ophthalmologists to screen the diabetes patients more efficiently is a potential solution to this problem [3]. DR is generally divided into non-proliferative DR (NPDR) and proliferative DR (PDR), occurs when diabetes affects the blood flow to the retina, damages the tiny blood vessels inside the retina causing leakage of blood and fluid on the surface of retina. Micro aneurysms (MA's) are the first sign of NPDR and are caused due to rupture in retinal capillaries and appear as small round dark red dots. The next most important sign of DR is haemorrhages (HA's). When MA's is sufficiently weekend, it may rupture and give rise to a HA's. Dot HA's and blot HA's appears as bright small and large red lesions [4].
In this present work to detect HA's, pre-processing is performed using median filter to remove noise and uneven illumination. The improved Toboggan based segmentation is used to locate and detect HA's in the retinal image. Based on the assumptions pixels that belong to HA's is irregular and complex structure having two similarities first it is red in colour, then its spatial location, the image is partitioned into non-overlapping super pixel of similar intensity covering the entire image [5]. Direct application of Toboggan segmentation algorithm leads to over segmentation due to noise or irregularities of gradient magnitude image [6]. A solution to the problem is existing in two steps. Due to this variability in the appearance of HA's, localization of contrast boundaries in gradient magnitude of contrast enhanced image for separating blood vessel and retinal background. Next, the maximum of gradient value is used to perform toboggan segmentation to segment pre-retinal HA's and sub-retinal HA's [7]. In literature reviewed methods, HA's have been segmented in addition to vessels by calculating difference between smoothed images and threshold output of pre-processed retinal image [8]. Evaluation of pixel density in threshold image to segment HA's carried out over 60 images from local database [9]. Colour normalization, two dimensional PCA and SVM were carried out in background retinopathy affected images as preprocessing and post-processing step to detect HA's [10].

\section{Materials and Methods}

A set of 81 fundus photograph with 31 normal and 50 abnormal images from publicly available STARE database, which were acquired using Topcon TRV-50 fundus camera. The images were captured using 24 bits per pixel at $605 \times 700$ pixels with a $35^{\circ} \mathrm{FOV}[11]$. 


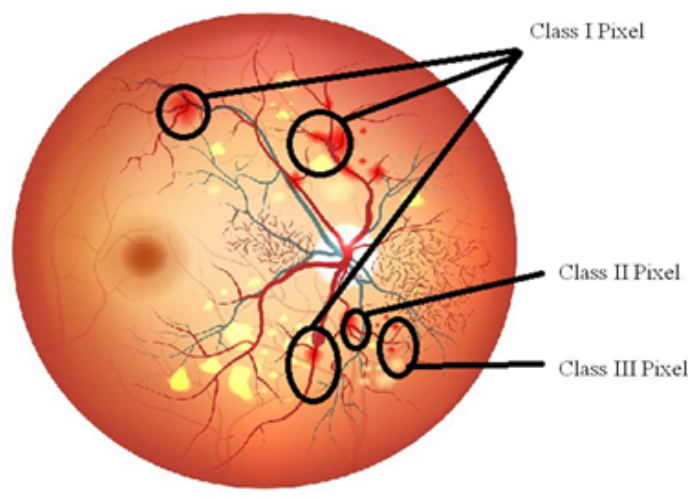

Figure 1. Illustration of tobaggan segmentation.

On the proposed work evaluation of automated DR detection system shows that an important cause of false negatives, as high as $50 \%$ is formed by images that contain only large HA's. Large number of HA's indicated more severity in DR detection, and improved detection of HAs will lead to elimination of more severe false negatives. In the retinal image RGB to grey conversion is performed and median filter is applied to make the image more visually appealing and remove salt and pepper noise. The entire retinal image is partitioned into nonoverlapping segments of similar intensities. Super-pixel based representation is an image re-sampling algorithm convert an image to irregular grid. At pixel level segmentation distribution of HA's pixel and non-HA's pixel are imbalanced. Usually, subset of background pixel used for training image re-sampling methods, super-pixel based approach maximizes diversity of training samples by retaining all important features [7]. Figure 1 shows the illustration of toboggan segmentation with class I, II and III pixels.

The following steps used to pre-process retinal image for HA segmentation:

Step 1: Image is converted from RGB to grey, median filter is a non-linear filter to preserve edge it is used.

Step 2: Apply Sobel operator to identify the edges and the magnitude of the gradient computed by

, Where the partial derivatives are computed by:
$\mathrm{S}_{\mathrm{x}}=\left(\mathrm{a}_{2}+\mathrm{ca}_{3}+\mathrm{a}_{4}\right)-\left(\mathrm{a}_{0}+\mathrm{ca}_{1}+\mathrm{a}_{6}\right)(1)$

$\mathrm{S}_{\mathrm{y}}=\left(\mathrm{a}_{0}+\mathrm{ca}_{1}+\mathrm{a}_{2}\right)-\left(\mathrm{a}_{6}+\mathrm{ca}_{5}+\mathrm{a}_{4}\right)(2)$

With the constant $c=2$. Like the other gradient operators, $S_{x}$ and $\mathrm{S}_{\mathrm{y}}$ can be implemented using convolution masks.

\begin{tabular}{cccccc}
\hline & $S_{x}$ & & $S_{y}$ & \\
-1 & 0 & 1 & 1 & 2 & 1 \\
-2 & 0 & 2 & 0 & 0 & 0 \\
1 & 0 & 1 & -1 & -2 & -1 \\
\hline
\end{tabular}

\section{Algorithm for toboggan approach to segment HA's [5]}

Step 1. Simulation of Sliding: This step records the sliding directions for all the class-I and class-II pixels in Figure 1.

Step 1.1. Simulate sliding for each of the class-I pixels in retinal image by recording its lowest neighbour's in a sliding list. Class I pixels used as seed pixel.

Step 1.2. Simulate keep-sliding for all class-II pixels in retinal image by region growing from class-I pixels.

Step 2. Label assignment for all the class-III pixels: Label all the local-minimum points (i.e., the entire bottom levels of catchment basins).

Step 3. Tobogganing Step: Assign label to each unlabelled pixel (i.e., class-I and class-II pixels) by first tobogganing and then backtracking, using best first search. Figure 2 shows the sample results of segmentation algorithm in retinal images.

\section{Results and Discussion}

Segmentation of HA's was performed by brightness correction method showed an SE of $80 \%$ and SPE of $88 \%$ [8]. Multi-level candidate extraction method was yielded an ACC of 94\% [9]. Combined 2 DPCA and virtual SVM were carried out to classify red lesions evaluated on 30 images from Singapore national eye centre [10]. In the present work, tobaggan segmentation applied on STARE database yielded an SE of $90.02 \%$, an SPE of $88.43 \%$, an ACC of $93.21 \%$ and less computation time of $0.28 \mathrm{~s}$. Table 1 shows the comparison of HA's segmentation algorithm with literature reviewed methods.

Table 1. Comparison of HA's segmentation algorithm with literature reviewed methods.

\begin{tabular}{|c|c|c|c|c|c|c|c|c|c|c|}
\hline \multirow[t]{2}{*}{ Author name } & \multirow[t]{2}{*}{ Citation } & \multirow[t]{2}{*}{ Algorithm } & \multirow{2}{*}{\multicolumn{2}{|c|}{ Database }} & \multirow{2}{*}{$\begin{array}{l}\text { No. } \\
\text { images }\end{array}$} & \multirow[t]{2}{*}{ of } & \multirow{2}{*}{$\begin{array}{l}\text { SE } \\
(\%)\end{array}$} & \multirow{2}{*}{$\begin{array}{l}\text { SPE } \\
(\%)\end{array}$} & \multirow{2}{*}{$\begin{array}{l}\text { ACC } \\
(\%)\end{array}$} & \multirow[t]{2}{*}{ Computation time(S) } \\
\hline & & & & & & & & & & \\
\hline Hatanaka et al. & [8] & Brightness correction & Own database & & 125 & & 80 & 88 & - & - \\
\hline Grisan et al. & [9] & $\begin{array}{l}\text { Local thresholding, Pixel } \\
\text { density }\end{array}$ & Own database & & 60 & & - & - & 94 & - \\
\hline Zhang et al. & {$[10]$} & $\begin{array}{l}\text { Color normalization, two } \\
\text { dimensional principal } \\
\text { component analysis, SVM }\end{array}$ & $\begin{array}{l}\text { Singapore } \\
\text { Eye Centre }\end{array}$ & National & 30 & & - & - & - & - \\
\hline
\end{tabular}




\begin{tabular}{|c|c|c|c|c|c|c|c|}
\hline $\begin{array}{l}\text { Proposed } \\
\text { method }\end{array}$ & Toboggan segmentation & STARE & 100 & 90.02 & 88.43 & 93.21 & 0.28 \\
\hline
\end{tabular}

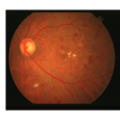

(a)

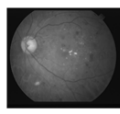

(b)

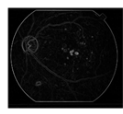

(c)

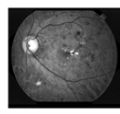

(d)

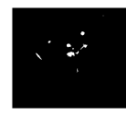

(c)

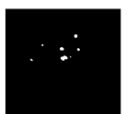

(f)
Figure 2. Sample result show segmentation of haemorrhages using toboggan algorithm (a) Original image; (b) Pre-processed image; (c) Gradient magnitude detection; (d) Image enhancement; (e) Toboggan segmentation; (f) Haemorrhages detection.

\section{Conclusion}

The novelty of the work is the application of improved toboggan segmentation for different kinds of haemorrhages like pre-retinal, sub-retinal and small/blot HA's with low/high density has regional/non-regional/not-crossing horizontal meridian characteristics. The main contribution of this technique is in the detection of retinal, pre-retinal, sub-retinal haemorrhage using a same pre-processing and segmentation technique. The proposed method obtained $90.02 \%$ in sensitivity, $88.43 \%$ in specificity and $93.21 \%$ in accuracy for the Indian eye database. Despite these results not being most favourable, they are encouraged and disclose that enhancements may be made.

\section{References}

1. Zaki WM, Zulkifley MA, Hussain A, Halim WH, Mustafa NB, Ting LS. Diabetic retinopathy assessment: Towards an automated system. Biomed Signal Processing Control 2016; 24: 72-82.

2. Figueiredo IN, Kumar SU, Oliveira CM, Ramos JD, Engquist B. Automated lesion detectors in retinal fundus images. Computers Biol Med 2015; 66: 47-65.

3. Sopharak A, Uyyanonvara B, Barman S. Simple hybrid method for fine micro aneurysm detection from non-dilated diabetic retinopathy retinal images. Comput Med Imaging Graphics 2013; 37: 394-402.
4. Akram MU, Khalid S, Khan SA. Identification and classification of micro aneurysms for early detection of diabetic retinopathy. Pattern Recognit 2013; 46: 107-116.

5. Fairfield J. Toboggan contrast enhancement for contrast segmentation. IEEE 1990; 1: 712-716.

6. Gonzalez RC, Woods RE. Image processing. Digital image processing. 2009; 2.

7. Tang L, Niemeijer M, Reinhardt JM, Garvin MK, Abramoff MD. Splat feature classification with application to retinal haemorrhage detection in fundus images. IEEE Trans Med Imaging 2013; 32: 364-375.

8. Hatanaka Y, Nakagawa T, Hayashi Y, Kakogawa M, Sawada A, Kawase K, Hara T, Fujita H. Improvement of automatic haemorrhage detection methods using brightness correction on fundus images. Med Imaging 2008; 69153.

9. Sakthivel K, Jayanthiladevi A, Kavitha C. Automatic detection of lung cancer nodules by employing intelligent fuzzy cmeans and support vector machine. Biomed Res 2016.

10. Zhang X, Chutatape O. A SVM approach for detection of haemorrhage's in background diabetic retinopathy. IEEE Int Joint Confer 2005; 4: 2435-2440.

11. Hoover AD, Kouznetsova V, Goldbaum M. Locating blood vessels in retinal images by piecewise threshold probing of a matched filter response. IEEE Trans Med Imaging. 2000; 19: 203-210.

\section{*Corresponding to}

S. Karkuzhali

Department of Information Technology

National Engineering College

Tamil Nadu

India 